This is an open access chapter licensed under the Creative Commons BY-NC 4.0 International License.

\title{
Annex 2: Inventory of stakeholder involvement techniques
}

Simone Casiraghi

Vrije Universiteit Brussel. E-mail: simone.casiraghi@vub.be. 


\begin{tabular}{|c|c|c|c|}
\hline 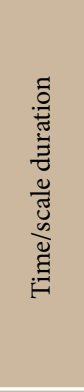 & 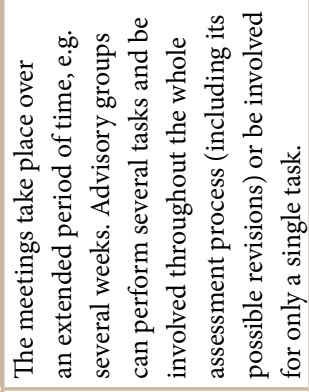 & 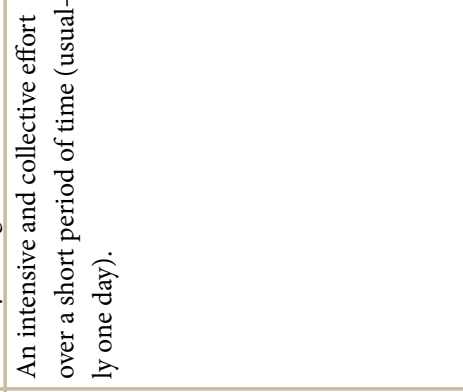 & 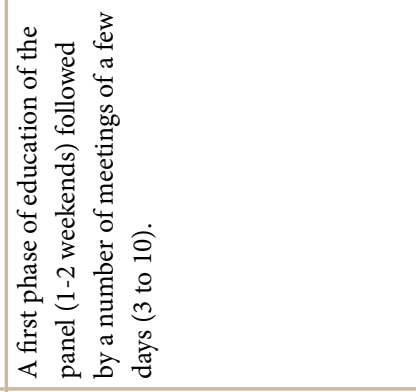 \\
\hline 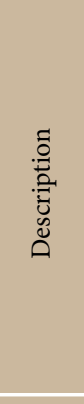 & 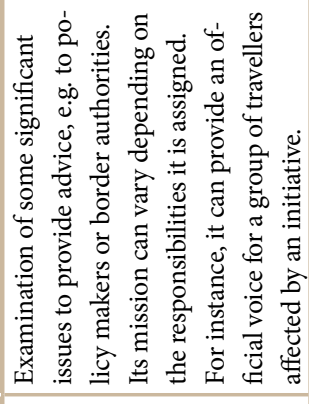 & 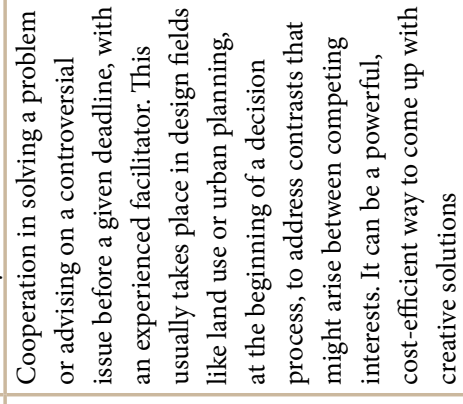 & 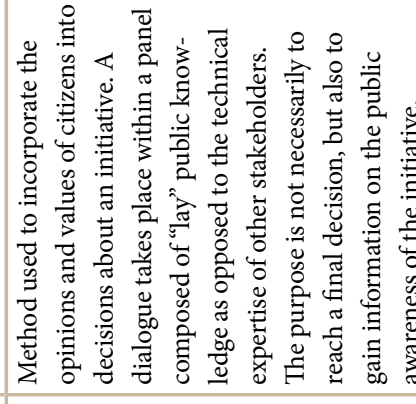 \\
\hline 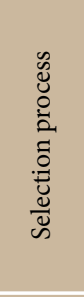 & 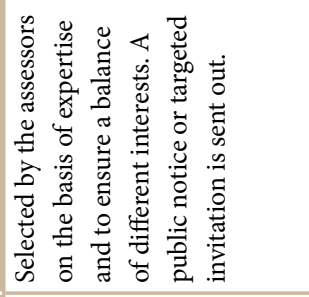 & 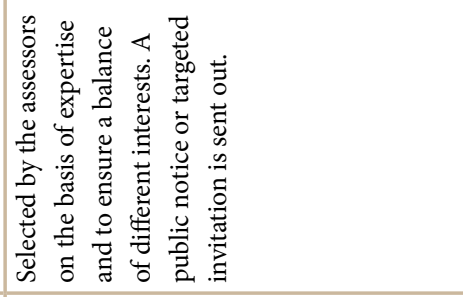 & 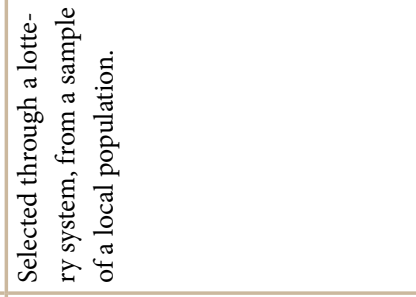 \\
\hline 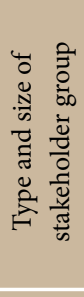 & 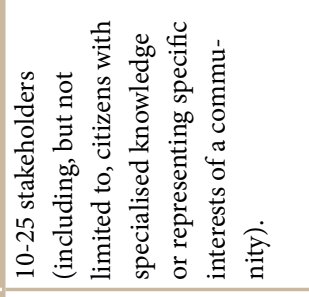 & 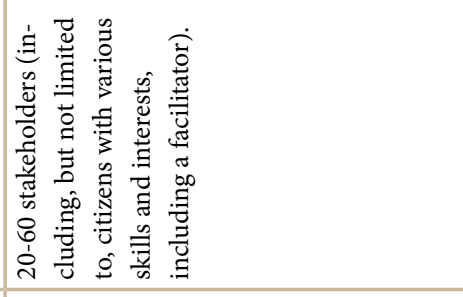 & 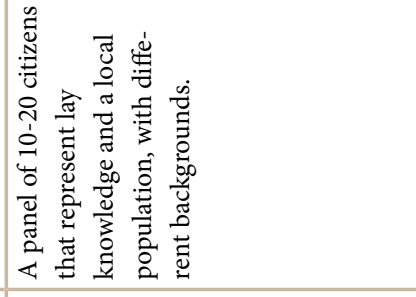 \\
\hline 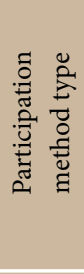 & 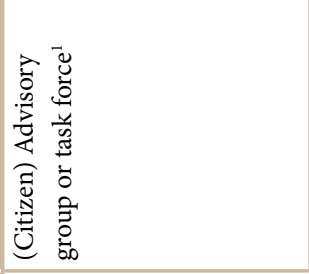 & 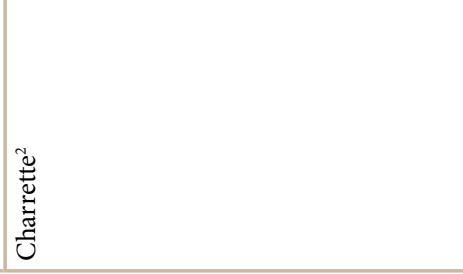 & 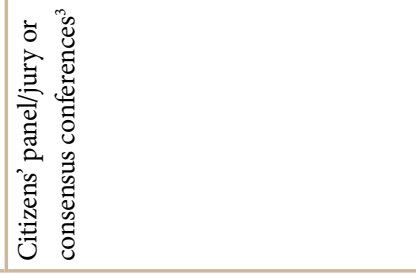 \\
\hline 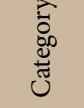 & \multicolumn{3}{|c|}{ 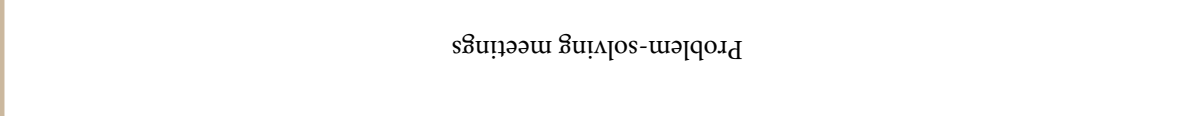 } \\
\hline
\end{tabular}


Annex 2

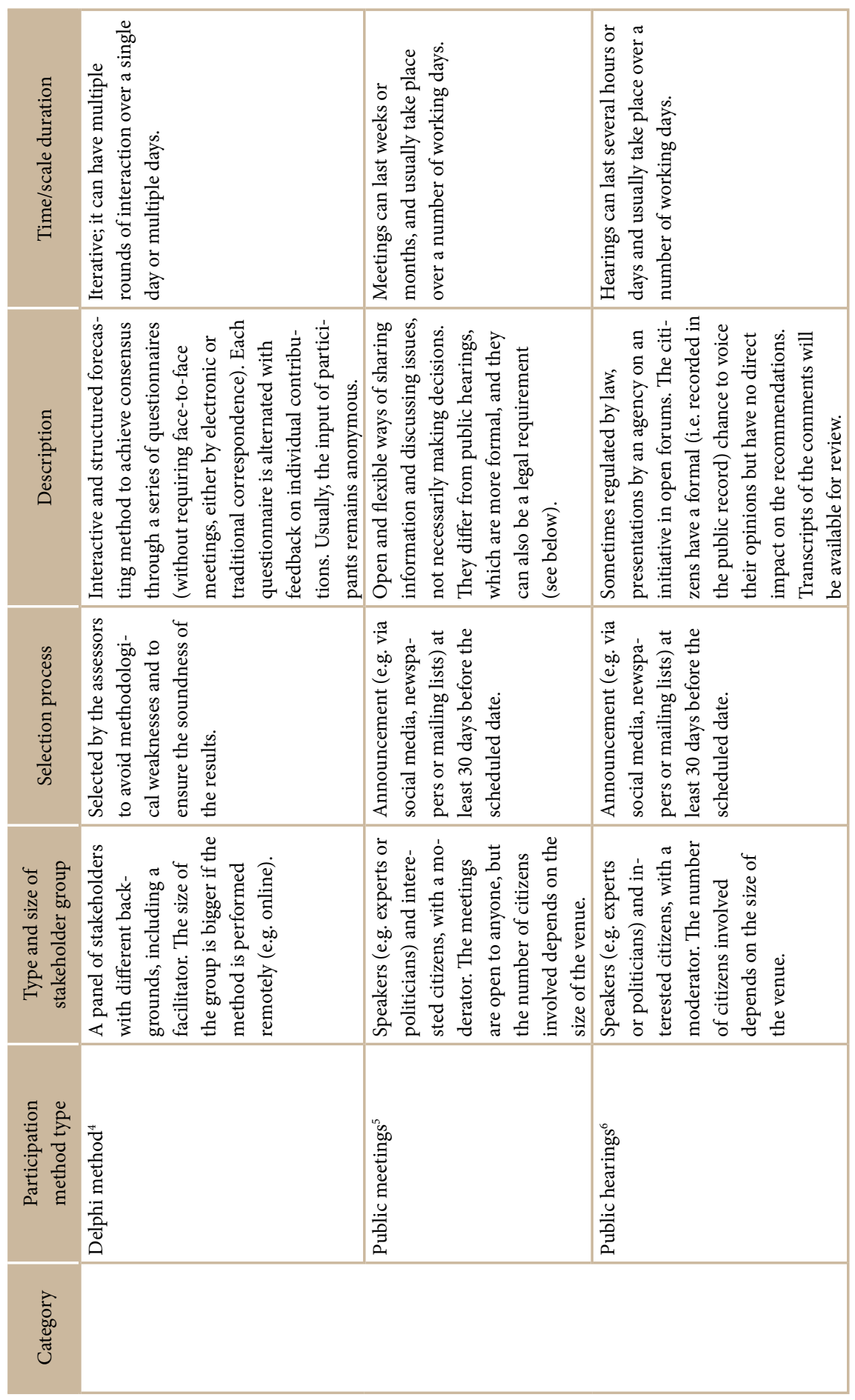




\begin{tabular}{|c|c|c|c|}
\hline 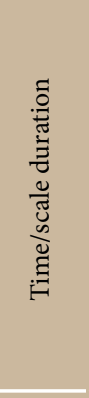 & 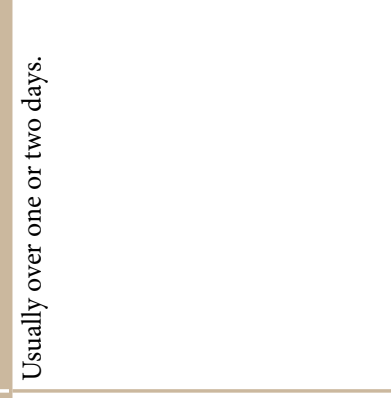 & 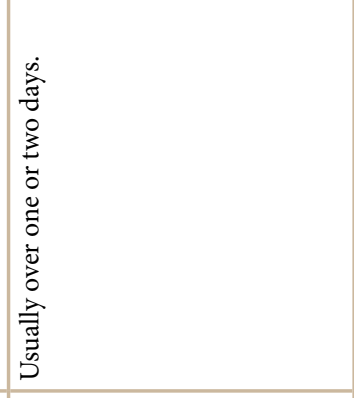 & 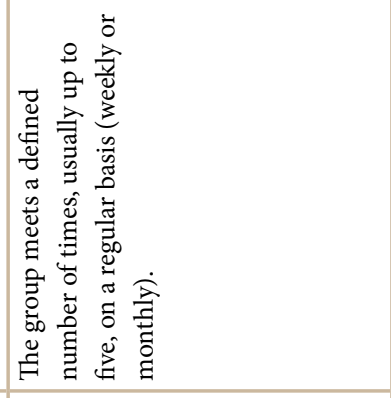 \\
\hline 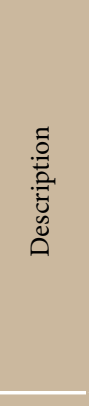 & 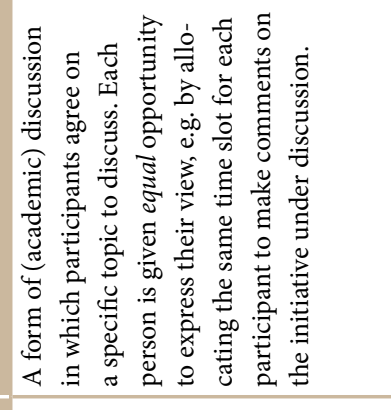 & 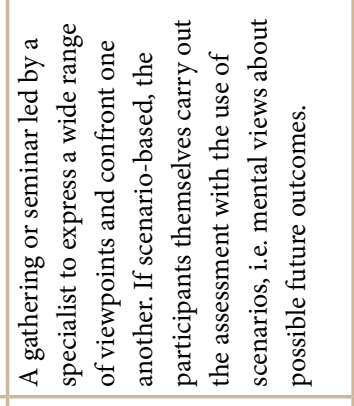 & 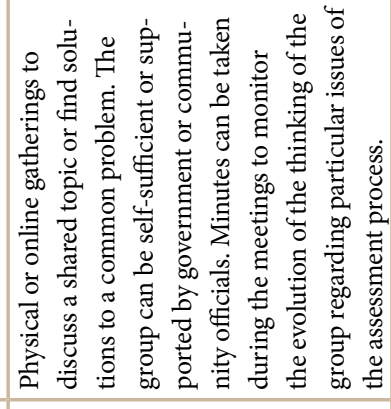 \\
\hline 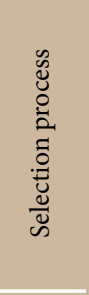 & 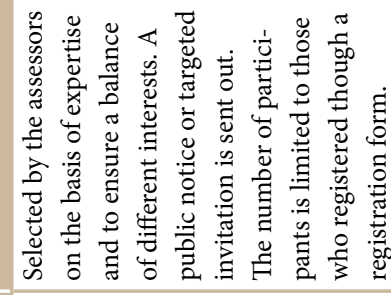 & 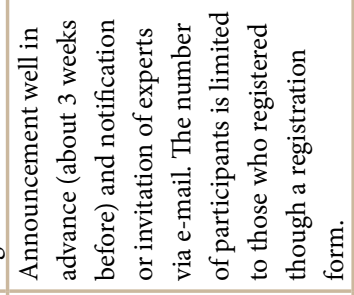 & 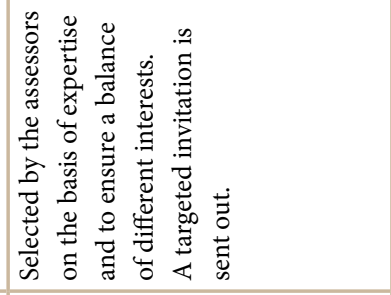 \\
\hline 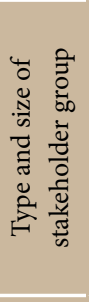 & 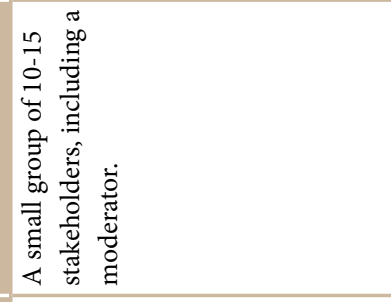 & 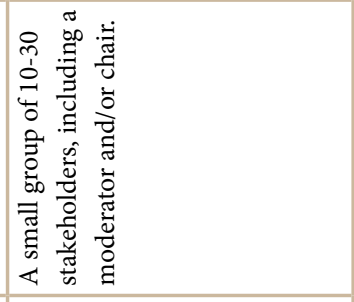 & 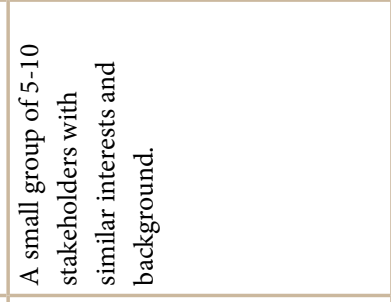 \\
\hline 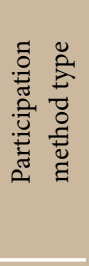 & 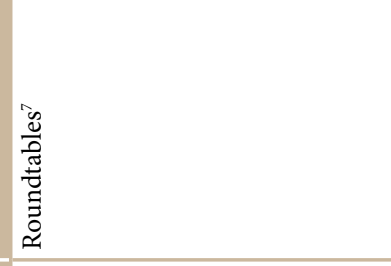 & 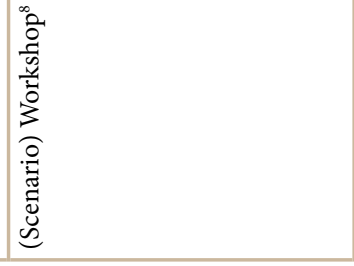 & 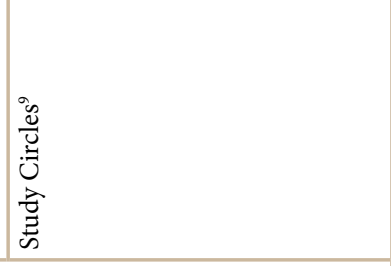 \\
\hline : & & & \\
\hline
\end{tabular}


Annex 2

\begin{tabular}{|c|c|c|c|c|}
\hline 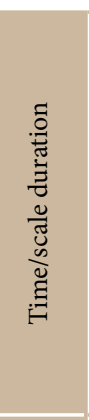 & 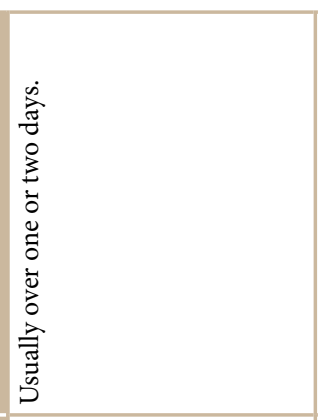 & 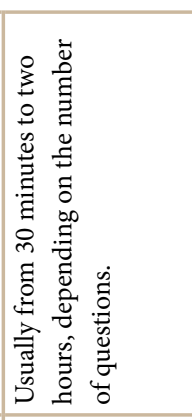 & 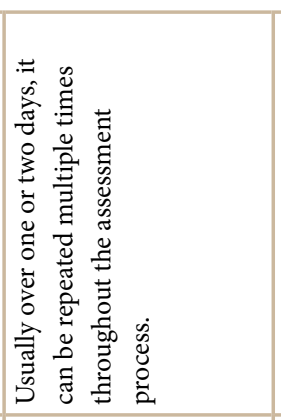 & 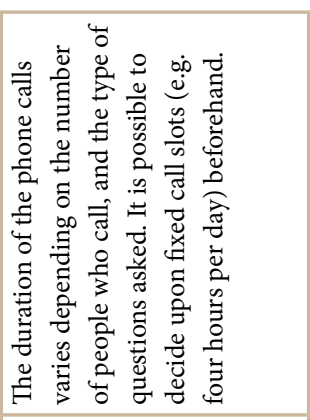 \\
\hline 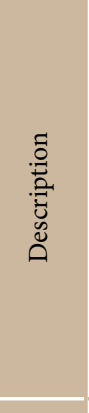 & 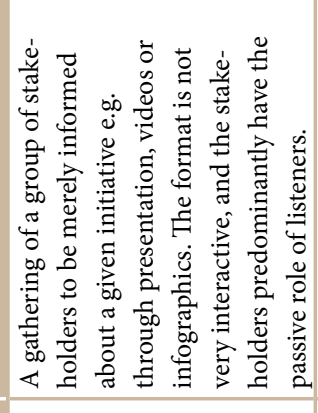 & 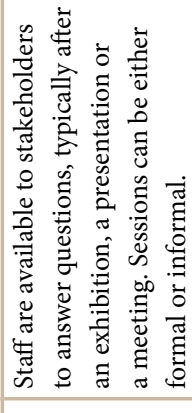 & 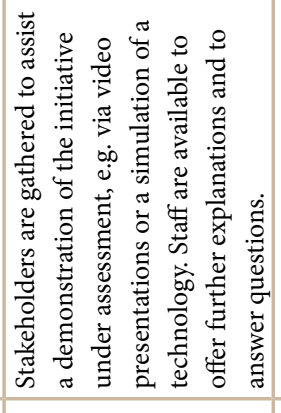 & 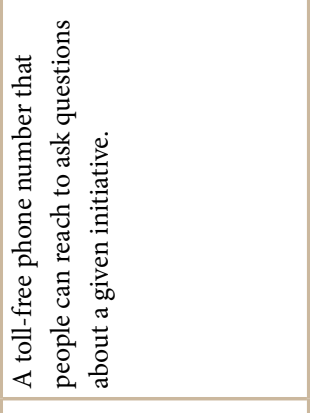 \\
\hline 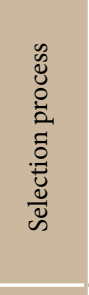 & 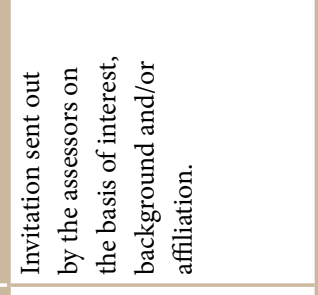 & 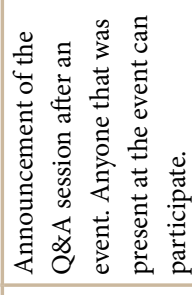 & 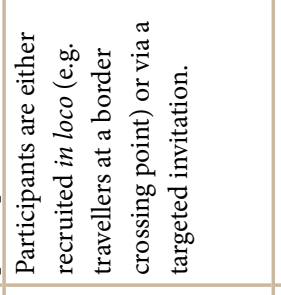 & 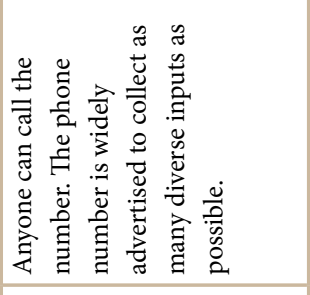 \\
\hline 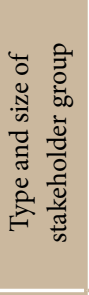 & 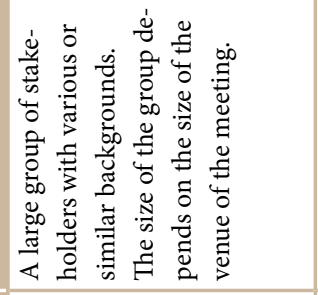 & 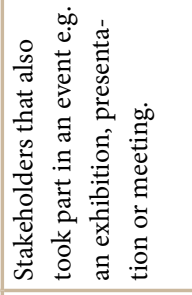 & 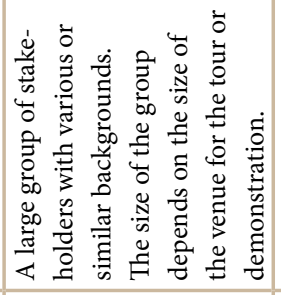 & 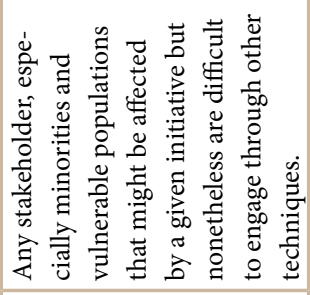 \\
\hline 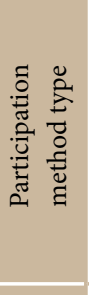 & 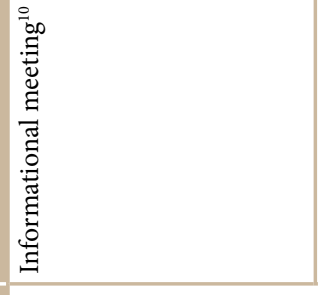 & 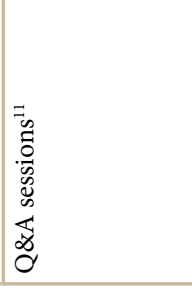 & 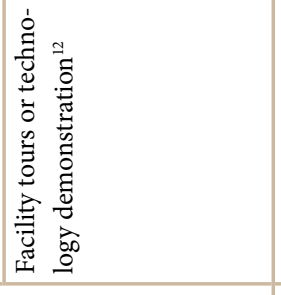 & 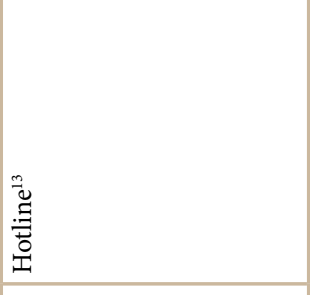 \\
\hline 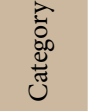 & \multicolumn{3}{|c|}{ 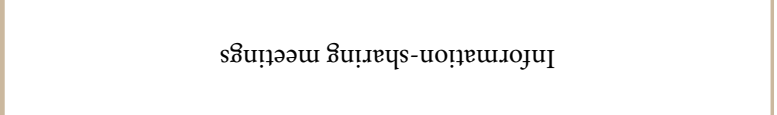 } & 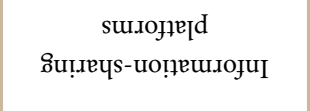 \\
\hline
\end{tabular}


Border Control and New Technologies

\begin{tabular}{|c|c|c|c|}
\hline 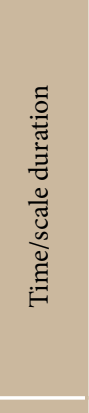 & 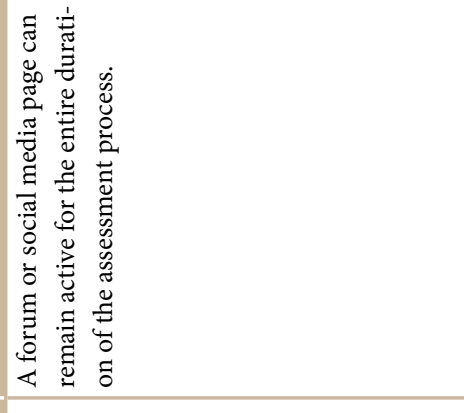 & 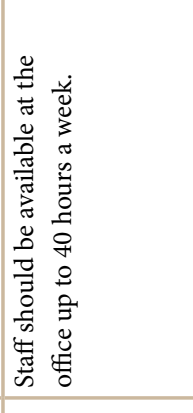 & 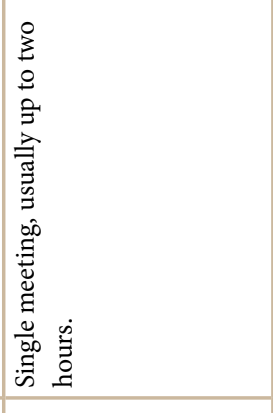 \\
\hline 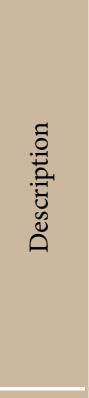 & 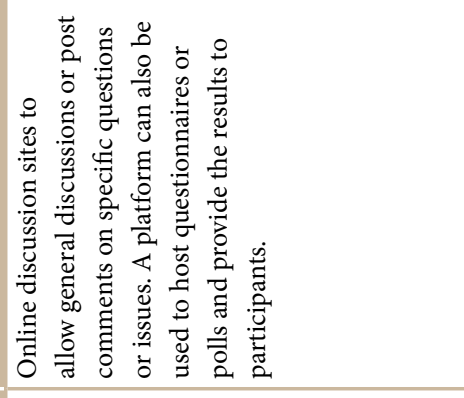 & 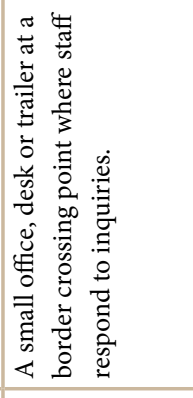 & 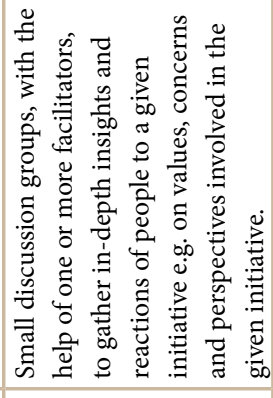 \\
\hline 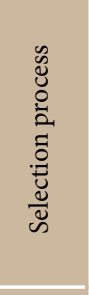 & 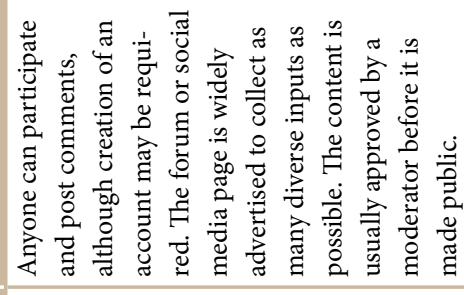 & 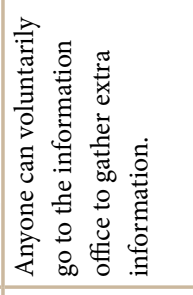 & 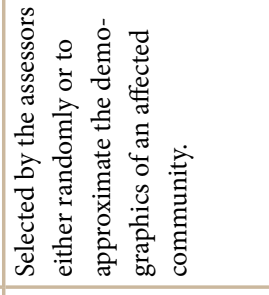 \\
\hline 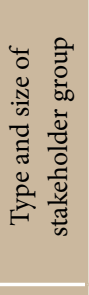 & 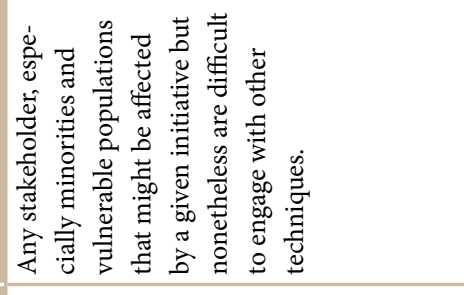 & 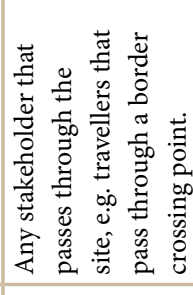 & 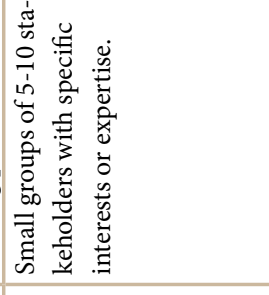 \\
\hline 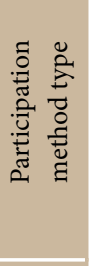 & 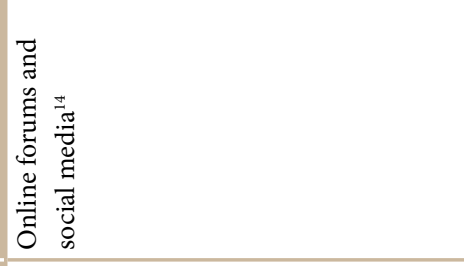 & 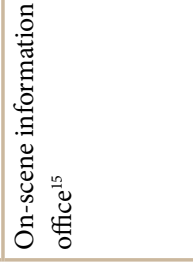 & 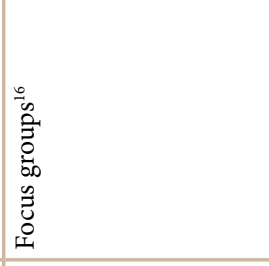 \\
\hline 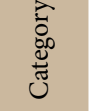 & & & słas.nns \\
\hline
\end{tabular}


Annex 2

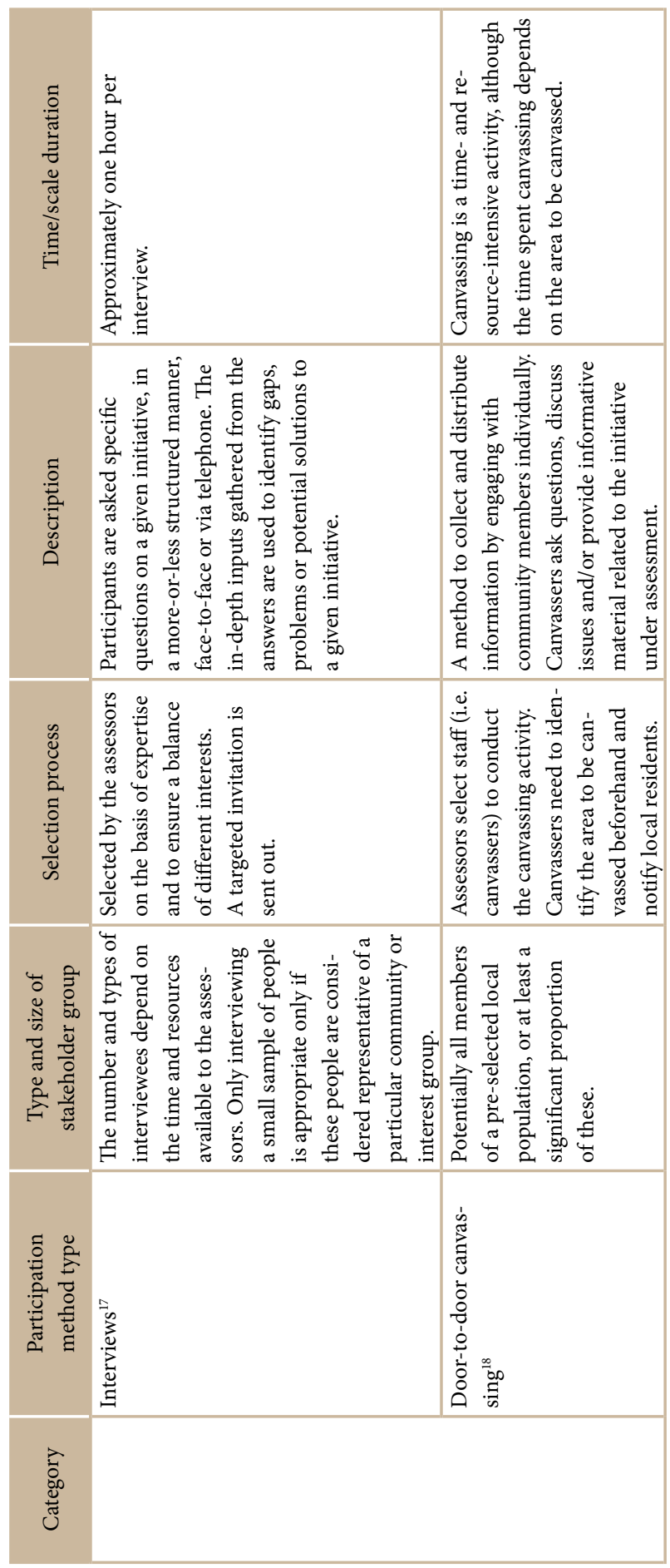




\begin{tabular}{|c|c|c|c|}
\hline 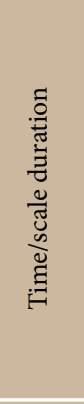 & 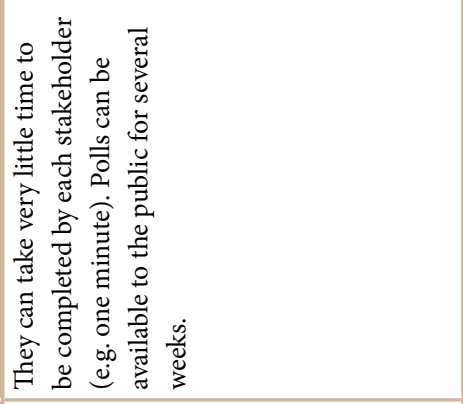 & 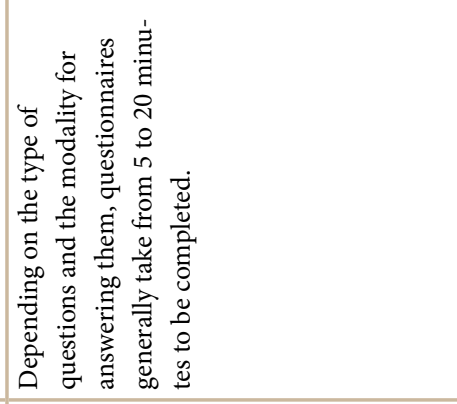 & 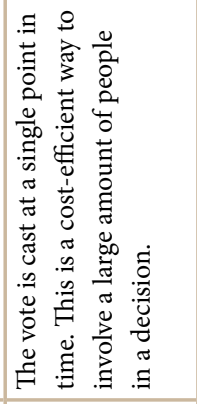 \\
\hline 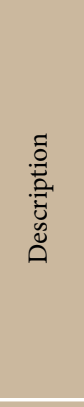 & 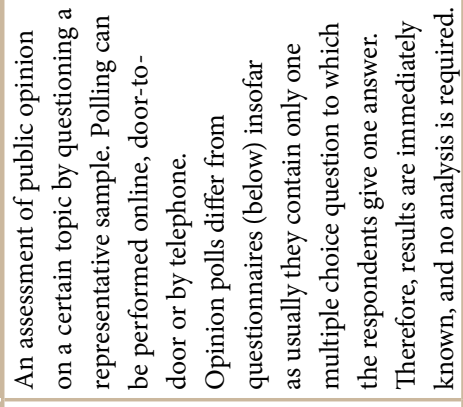 & 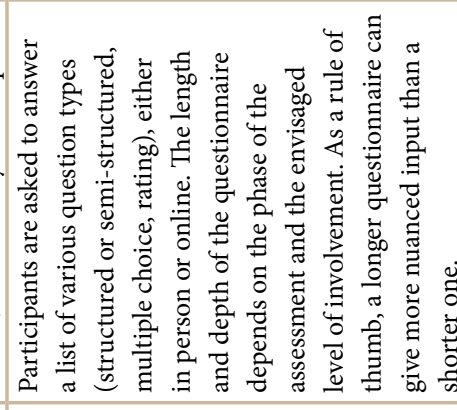 & 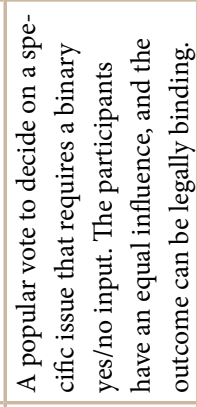 \\
\hline 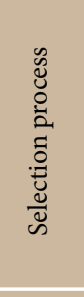 & 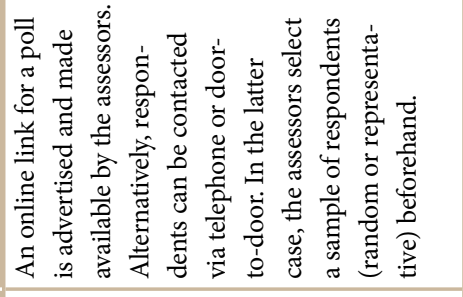 & 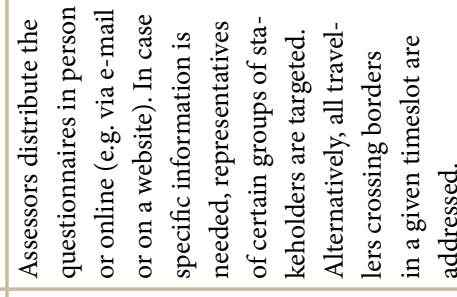 & 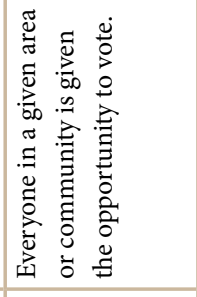 \\
\hline 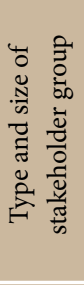 & 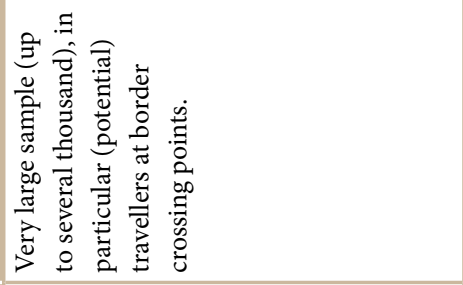 & 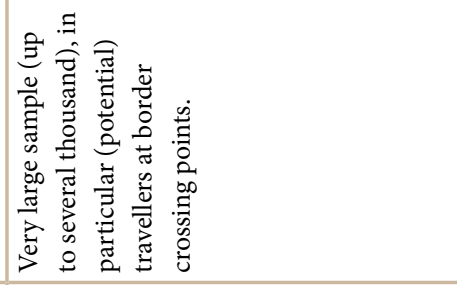 & 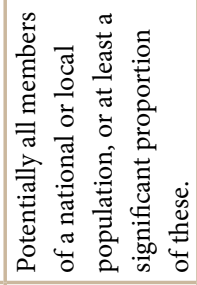 \\
\hline 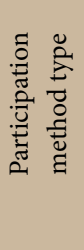 & 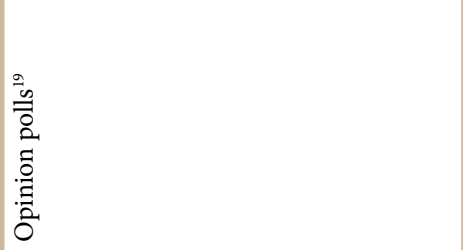 & 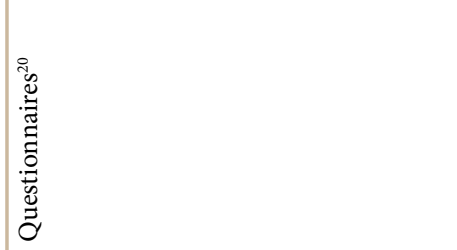 & 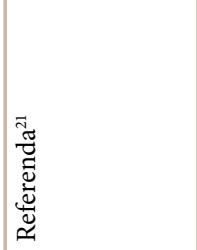 \\
\hline 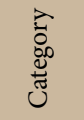 & & & \\
\hline
\end{tabular}




\section{Endnotes}

1. James L. Creighton, The Public Participation Handbook: Making Better Decisions through Citizen Involvement (San Francisco: Jossey-Bass, 2005), 183; Gene Rowe and Lynn J. Frewer, "Public Participation Methods: A Framework for Evaluation," Science Technology and Human Values 25, no. 1 (2000): 3-29, https://doi.org/10.1177/016224390002500101; US Environmental Protection Agency, RCRA Public Participation Manual, 1996.

2. James A. Segedy and Bradley Johnson, The Neighbourhood Charrette Handbook, 2004, https://www.michigantownships.org/downloads/charrette_handbook_2.pdf; Gail Lindsey, Joel Ann Todd, and Sheila J. Hayter, A Handbook for Planning and Conducting Charrettes for High-Performance Projects, 2009, https://www.nrel.gov/docs/fy03osti/33425.pdf; Organization for Economic Co-operation and Development (OECD), Stakeholder Involvement Techniques: Short Guide and Annotated Bibliography, Nuclear Energy Agency, 2004, 40.

3. Ned Crosby, Janet M. Kelly, and Paul Schaefer, "Citizens Panels: A New Approach to Citizen Participation," Public Administration Review 46, no. 2 (1986): 170; Simon Joss and John Durant, Public Participation in Science. The Role of Consensus Conference in Europe (London: Science Museum, 1995).

4. Harold Linstone and Murray Turoff, The Delphi Method: Techniques and Applications (Boston, MA: Addison Wesley Publishing Company, 1975); Gene Rowe and George Wright, "The Delphi Technique as a Forecasting Tool: Issues and Analysis," International Journal of Forecasting 15, no. 4 (1999): 353-75.

5. US Environmental Protection Agency, RCRA Public Participation Manual, 117; Creighton, The Public Participation Handbook: Making Better Decisions through Citizen Involvement, 130.

6. Rowe and Frewer, "Public Participation Methods: A Framework for Evaluation"; US Environmental Protection Agency, RCRA Public Participation Manual, 123; Creighton, The Public Participation Handbook: Making Better Decisions through Citizen Involvement, 130.

7. Organization for Economic Co-operation and Development (OECD), Stakeholder Involvement Techniques: Short Guide and Annotated Bibliography, 31; Stefan Taschner and Matthias Fiedler, "Stakeholder Involvement Handbook," 2009, 20; Organization for Economic Co-operation and Development (OECD), Stakeholder Involvement Techniques: Short Guide and Annotated Bibliography, http://www.aeneas-project.eu/docs/AENEAS_StakeholderInvolvementHandbook.pdf.

8. Gill Ringland, "The Role of Scenarios in Strategic Foresight," Technological Forecasting and Social Change 77, no. 9 (2010): 1493-98, https://doi.org/10.1016/j.techfore.2010.06.010; US Environmental Protection Agency, RCRA Public Participation Manual, 134.

9. Organization for Economic Co-operation and Development (OECD), Stakeholder Involvement Techniques: Short Guide and Annotated Bibliography, 31.

10. Taschner and Fiedler, "Stakeholder Involvement Handbook," 20.

11. US Environmental Protection Agency, RCRA Public Participation Manual, 106.

12. US Environmental Protection Agency, 56.

13. Creighton, The Public Participation Handbook: Making Better Decisions through Citizen Involvement, 118; US Environmental Protection Agency, RCRA Public Participation Manual, 100.

14. Creighton, The Public Participation Handbook: Making Better Decisions through Citizen Involvement, 119.

15. US Environmental Protection Agency, RCRA Public Participation Manual, 103. 
16. Jay Klagge, Guidelines for Conducting Focus Groups, 2018, https:/doi.org/10.13140/ RG.2.2.33817.47201; Gloria E. Bader and Catherine A. Rossi, Focus Groups. A Step by Step Guide (San Diego, CA: The Bader Group, 2002); US Environmental Protection Agency, RCRA Public Participation Manual, 77.

17. Creighton, The Public Participation Handbook: Making Better Decisions through Citizen Involvement, 190; US Environmental Protection Agency, RCRA Public Participation Manual, 70.

18. US Environmental Protection Agency, RCRA Public Participation Manual, 80.

19. Louis M. Rea and Richard A. Parker, Designing and Conducting Survey Research. A Comprehensive Research Guide (Jossey-Bass, 2005); US Environmental Protection Agency, RCRA Public Participation Manual, 90; Creighton, The Public Participation Handbook: Making Better Decisions through Citizen Involvement, 128.

20. Rea and Parker, Designing and Conducting Survey Research. A Comprehensive Research Guide, 90; US Environmental Protection Agency, RCRA Public Participation Manual; Creighton, The Public Participation Handbook: Making Better Decisions through Citizen Involvement, 128.

21. Rowe and Frewer, "Public Participation Methods: A Framework for Evaluation," 19. 